TITLE:

\title{
Anthocyanins in perilla plants and dried leaves
}

\section{$\operatorname{AUTHOR}(\mathrm{S})$ :}

Fujiwara, Yumi; Kono, Miya; Ito, Airi; Ito, Michiho

\section{CITATION:}

Fujiwara, Yumi ...[et al]. Anthocyanins in perilla plants and dried leaves. Phytochemistry 2018, 147: 158-166

\section{ISSUE DATE:}

2018-03

URL:

http://hdl.handle.net/2433/244233

\section{RIGHT:}

(C) 2018. This manuscript version is made available under the CC-BY-NC-ND 4.0 license

http://creativecommons.org/licenses/by-nc-nd/4.0/.; The full-text file will be made open to the public on 3 March 2020 in accordance with publisher's 'Terms and Conditions for Self-Archiving'.; この論文は出版社版でありません。引用の際 には出版社版をご確認ご利用ください。; This is not the published version. Please cite only the published version. 
Anthocyanins in perilla plants and dried leaves

Yumi Fujiwara a, b, Miya Kono a, Airi Ito a, Michiho Ito a*

Affiliations

a Department of Pharmacognosy, Graduate School of Pharmaceutical Science, Kyoto

University; 46-29 Yoshida-Shimo-Adachi-cho, Sakyo-ku, Kyoto 606-8501, Japan.

b Department of Pharmacognosy, College of Pharmacy, Kinjo Gakuin University; 2-1723

Ohmori, Moriyama-ku, Nagoya, Aichi 463-8521, Japan.

Corresponding author

* Michiho Ito, Department of Pharmacognosy, Graduate School of Pharmaceutical

Science, Kyoto University; 46-29 Yoshida-Shimo-Adachi-cho, Sakyo-ku, Kyoto 606-

8501, Japan. E-mail: michihoi@pharm.kyoto-u.ac.jp. Phone: +81 75753 4507. Fax:+81

757534507. 


\section{Abstract}

High-quality perilla leaves are purple on upper and lower surfaces and have a good aroma. The Japanese Pharmacopoeia specifies the content of essential oils in perilla leaves but not that of anthocyanins. Several reports have described the chemical species of anthocyanins in red perilla, but a complete analysis of anthocyanins in perilla has not been reported. In this study, the anthocyanins in the leaves of cultivated and wild species of perilla and those in commercially available perilla herbs were studied. Red perilla and most $P$. citriodora strains accumulate cyanidin derivatives that differ in the acyl group on the glucose moiety at the $3-\mathrm{O}$ and 5-O-positions of the anthocyanins. Several strains of $P$. citriodora contain cyanidin derivatives that are different from those in red perilla and most $P$. citriodora species. Green perilla and wild species other than P. citriodora do not contain foliar anthocyanins. The anthocyanins in commercially available perilla herbs and natural dyes made from red perilla were in agreement with those in fresh red perilla leaves and most $P$. citriodora samples. The amounts and types of anthocyanins were not associated with place of cultivation, although some changes occurred due to degradation during storage. These results provide clues regarding the biosynthesis of anthocyanins in perilla and the evolution of red perilla. The characteristics and stability of anthocyanins are discussed.

Keywords: Perilla frutescens; Labiatae; Anthocyanin; Cyanidin; HPLC analysis; Biosynthetic pathway; Acyltransferase; 5- $O$-glucosyltransferase specific to cyanidin 3$O$ glucoside 


\section{Introduction}

Perilla frutescens is an annual plant used as a fresh herb in cooking. Purple perilla leaves are used pharmaceutically to ease stomach disorders and induce sweating (Ministry of Health, Labour and Welfare, 2016), and are blended in several Japanese traditional medicinal formulations such as Kososan, Shimpito, and Hangekobokuto. According to Honzokomoku, an ancient Chinese textbook of medicinal plants, highquality perilla leaves are purple on both sides and have a good aroma (Shirai, 1930).

The reddish color of perilla leaves is due to anthocyanin pigments. Anthocyanins have antioxidant activity, and natural materials containing these compounds are often used as ingredients in health foods. Anthocyanins are also used as natural food coloring, which most consumers prefer to synthetic food coloring. Specifically, anthocyanins and other dyes of plant origin are used to impart red, purple, or blue colors to food. Perilla has traditionally been used to color pickles and drinks in Japan, and food dyes made from red perilla have recently become available as commercial products. However, anthocyanins in perilla leaves are unstable and are easily damaged by high $\mathrm{pH}$, light, heat, and ionized compounds (Goto et al., 1976; Yoshida et al., 1990). Several studies have identified some of the anthocyanins in red perilla, but a comprehensive analysis has not been reported.

In Japan, there are four Perilla species: a cultivated species, $P$. frutescens, and three wild species, P. citriodora, P. hirtella, and P. setoyensis. Each has unique characteristics and the number of chromosomes differs between the cultivated species $(2 n=40)$ and the wild species $(2 n=20)$. The results of our previous experiments on genetic crossing and DNA polymorphisms suggest that $P$. frutescens is an amphidiploid of two wild species and that $P$. citriodora may be one of the original species (Ito et al., 1998). $P$. citriodora leaves are red on the abaxial side but not on the adaxial side. It would be interesting to elucidate the mechanism controlling the red color of perilla leaves. In 
this study, the anthocyanins in the leaves of cultivated and wild species of perilla, as well as those in commercially available perilla herbs, were identified. In addition, a possible biosynthetic pathway for anthocyanins is postulated from the chemical species of anthocyanins, and the chemotaxonomical implications of our findings are discussed.

\section{Results and Discussion}

\subsection{HPLC analysis of anthocyanins in fresh and dried perilla leaves and in natural} dyes made from perilla

The HPLC patterns of samples of different perilla strains were different and could be divided into four groups (Fig. 1). A maximum of seven peaks are present in each HPLC pattern. All red perilla samples ( $P$. frutescens), most $P$. citriodora samples, and commercially available perilla herbs and natural dyes made from red perilla provided pattern A (five peaks, Nos. 3 to 7). Only one strain of P. citriodora (Strain No. 87) showed pattern B (seven peaks, Nos. 1 to 7), and another strain of P. citriodora (Strain No. 5717) showed pattern C (two peaks, Nos. 1 and 2). In contrast, most green perilla ( $P$. frutescens) samples, as well as $P$. setoyensis and $P$. hirtella samples, provided no anthocyanin peaks (pattern D).

\subsection{Identification of anthocyanins}

Anthocyanins in perilla could be identified by comparing their retention times with those of authentic compounds; however, some of the authentic anthocyanins were not available. The MS and NMR spectra of these anthocyanins were examined to determine their aglycone structures. Anthocyanidins found in plant anthocyanins are classified as pelargonidin, cyanidin, delphinidin, peonidin, petunidin, and malvidin, and they have different patterns of hydroxyl or methoxy groups on the B-ring. The more hydroxyl groups an anthocyanidin has, the bluer it appears. Pelargonidin with 
one hydroxyl group is orange and has a maximum absorption at $515 \mathrm{~nm}$; cyanidin with two hydroxyl groups is reddish violet and has a maximum absorption at $528 \mathrm{~nm}$; and delphinidin with three hydroxyl groups is blue-violet and has a maximum absorption at $543 \mathrm{~nm}$ (Yokoi et al., 1979). Previous reports on perilla anthocyanidins showed that almost all the aglycones were cyanidin (Yoshida et al., 1990; Yamazaki et al., 2003), and cDNA clones encoding the enzymes involved in anthocyanin biosynthesis, namely, chalcone synthase, flavanone 3-hydroylase, dihydroflavonol 4-reductase, UDP glucose: flavonoid 3-O-glucosyltransferase, and flavonoid 3'-hydroxylase have been isolated from the leaves of a red perilla (Gong et al., 1997; Kitada et al., 2001). Therefore, the seven anthocyanidins found in perilla were expected to have cyanidin as an aglycone, and this was supported by the maximum absorption of $528 \mathrm{~nm}$ for the seven compounds. The compounds were further analyzed by MS and NMR to confirm their structures. Anthocyanins were extracted from red leaves of Strain No. 5717 (73.7 g) and Strain No. 5601 (34.5 g) and purified to single compounds. The yields of the compounds based on HPLC peak areas were as follows: peak 1, $32.1 \mathrm{mg}$; peak 2, $12.7 \mathrm{mg}$; peak 3, $13.7 \mathrm{mg}$; peak 4, $4.6 \mathrm{mg}$; peak 5, $5.8 \mathrm{mg}$; peak 6, $8.6 \mathrm{mg}$; and peak 7, $38.2 \mathrm{mg}$. Peaks 1 and 2 were purified from Strain No. 5717 and peaks 3, 4, 5, 6, and 7 were purified from Strain No. 5601. The compounds corresponding to peaks 1, 3, 4, 5, 6, and 7 were identified by MS and ${ }^{1} \mathrm{H}$ NMR analyses and are shown in Fig. 2 (Charron et al., 2007; He et al., 2010; Kondo et al., 1989; Yoshida et al., 1990). These compounds are cyanidin derivatives with different acyl groups on the glucose moiety attached to the anthocyanin 3-O and 5-O-positions. The MS data for peak 3 showed the co-existence of two compounds that were subsequently separated. The ${ }^{1} \mathrm{H}$ NMR data of peak 2 did not correspond to any previously reported anthocyanin and MS showed peaks at $\mathrm{m} / \mathrm{z}$ $697.16[\mathrm{M}]^{+}, 535.11[\text { cyanidin }+ \text { glucose }+ \text { malonyl }]^{+}, 449.11[\mathrm{Cy}+\mathrm{Glc}]^{+}$, and 287.05 $[\mathrm{Cy}]^{+}$. Perilla expresses enzymes that catalyze malonylation at the 6'- $O$-position of 5- $O$ 
glucose (Matsune et al., 1997; Suzuki et al., 2001). The ${ }^{1} \mathrm{H}$ NMR data of peak 2 were similar to those of cyanidin-3,5- $O$-diglucoside (Yoshida et al., 1990), but a proton signal corresponding to the 6'-C-position in glucose A was shifted downfield (data reported by Goto et al. (1976): 3.96, $3.75 \rightarrow$ data of peak $2: 4.55,4.34)$. This shift can be explained by the malonylation of glucose A. Other peak 2 signals shifted upfield and corresponding to glucose were similar to pelargonidin 3- $\mathrm{O}$-glucoside-5- $\mathrm{O}$ malonylglucoside, a compound malonylated at glucose $\mathrm{B}$ of pelargonidin 3,5- $\mathrm{O}$ diglucoside (Hosokawa et al., 1995). Peak 2 was thus identified as cyanidin $3-O$ glucoside-5- $O$-malonylglucoside. The ${ }^{13} \mathrm{C}$ NMR and ${ }^{1} \mathrm{H}$ NMR signals of peak 2 were assigned using 2D NMR and are shown in Table 3.

Previous studies on perilla anthocyanins have had conflicting results regarding the compounds present in red perilla leaves. For example, Yoshida et al. (1990) found compounds 1 and 3 to 7 and Yamazaki et al. (2003) found compounds 2, 3a, and 4 to 7 . No previous report is consistent with the compounds in the present report (compounds 3 to 7). This discrepancy is in part due to the degradation of anthocyanins: compound 1 in Yoshida's report and compound 2 in Yamazaki's report could be formed by the degradation of compounds 3 to 7 during extraction and storage prior to analysis. Compound $3 \mathrm{~b}$ is absent from Yamazaki et al.'s report because they only used HPLCPDA-MS for compound identification and thus could not separate compound $3 \mathrm{~b}$ from its isomer, compound 6 .

In summary, red perilla and most $P$. citriodora stains accumulate compounds $3,4,5$, 6, and 7, and two strains of P. citriodora (Strain Nos. 87 and 5717) produce the anthocyanins compounds 1 and 2. Green perilla and wild species of perilla other than P. citriodora do not contain foliar anthocyanins.

\subsection{Oil type and anthocyanin pattern are genetically independent}


Table 1 shows the anthocyanin pattern of fresh perilla leaves corresponding to Fig. 1 and their oil type. Perilla oils can be classified according to the main oil: perillaldehyde (PA), citral (C), elsholtziaketone (EK), perillaketone (PK), perillene (PL), shisofuran (SF), or piperitenone (PT). The main oil can then be grouped by monoterpene type: myristicin (PP-m), dillapiol + myristicin (PP-dm), elemicin + myristicin (PP-em), dillapiole + elemicin + myristicin (PP-dem), and dillapiol + myristicin + nothoapiol (PPdmn), which can themselves be grouped further by phenylpropene type (Ito et al., 1999a; Ito et al., 1999b; Ito et al., 2002). A series of genetic crossing experiments using established pure lines showed that perilla oil types are genetically controlled (Honda, 1996; Ito et al., 2002; Koezuka et al., 1986; Masumoto et al., 2010) and are independent of the genetic control of leaf color (Honda et al., 1990; Koezuka et al., 1985; Koezuka et al., 1986). This study further investigated the types of anthocyanins in red perilla and showed that the oil type and types of anthocyanins are determined independently. Pattern A anthocyanins include all but the SF type. In contrast, oil type $\mathrm{C}$ strains, namely, Strain Nos. 87, 4931, 5498, and 5717, showed type A, B, and C anthocyanins.

\subsection{Chemical compositions of anthocyanins in commercially available perilla herbs and} dye samples

All perilla herbs and natural dyes made from red perilla provided HPLC pattern A and contained compounds $3,4,5,6$, and 7 as anthocyanin species. However, dried leaves showed a wide range of concentrations of these compounds. Compound 7 comprised more than $60 \%$ of the total anthocyanins in fresh leaves, followed by equal amounts of compounds 6 and $4(8-17 \%)$, and compounds 5 and $3(2-5 \%)$. These ratios are in accordance with 16 strains of pattern A of pure lines. Commercially available dried leaves contain more of compound 3 than compound 4, in contrast with fresh leaves. Furthermore, some dried leaves contain mostly compound 6 , while compound 3 
is present in some dried leaves in amounts equal to or greater than the amounts of 4 and 5 combined. Fresh leaves, on the other hand, contain only trace amounts of compound 3. Differences between fresh and dried leaves are likely due to differences in place of cultivation and storage conditions. All fresh leaves were cultivated in Japan, whereas most of the dried leaves were cultivated in China over a wide area. Chemical compositions were compared between places of cultivation and were highly inconsistent. Harvest time and anthocyanin composition were also compared. Dried leaves were harvested between 2 and 56 months before analysis. The anthocyanin composition of freshly dried leaves resembled that of fresh leaves, whereas the amount of compound 7 decreased and that of compound 6 increased as the leaves aged (Fig. 3). Comparison of the chemical composition of the leaves by place of cultivation and harvest time indicates that these differences in the composition of dried and fresh leaves are likely due to the elimination of an acyl group; compound 6 can be formed by the degradation of compound 7 during storage prior to analysis. Dye samples had the highest level of compound 6 and the lowest level of compound 7 of all leaf samples, indicating that compound 7 was degraded by processing and during storage. Anthocyanins are unstable and are easily degraded by high $\mathrm{pH}$, light, heat, and salt (Goto et al., 1976; Yoshida et al., 1990). Several reports have examined the effects of anthocyanin extraction conditions (Goto et al., 1983; Kondo et al., 1989; Tamura et al., 1983). As noted in Section 2.2, different extraction procedures can alter the structure of anthocyanin, consistent with previous reports. Yu et al. (1997) investigated the degradation of compound 7 by HPLC analysis. When treated with $1 \% \mathrm{HCl}-\mathrm{MeOH}$, the methyl ester of compound 7 appeared after $1 \mathrm{~h}$ at room temperature and compound 7 disappeared after $24 \mathrm{~h}$ of treatment and was converted to compound 6 . In contrast, acidic conditions, such as treatment with aqueous trifluoroacetic acid (TFA) solution during anthocyanin extraction, do not convert compound 7 to other compounds. 
Therefore, compound 7 is converted to compound 6 by the elimination of a malonyl residue and this is inhibited by acidic conditions. Yoshida et al. reported the cis-trans isomerization of compounds 7 and 4 by irradiation of UV light or sunlight. Compound 7 and other anthocyanins that contain cinnamic acid residues isomerize when irradiated with UV light (Yoshida et al., 1990). Yu et al. noted that the photoisomerization may also dissipate the high energy of short-wavelength light. The stability of compounds 1 , $4,5,6$, and 7 in acidic aqueous solution at $50^{\circ} \mathrm{C}$ was examined by Yoshida et al. Compound 4 showed the same stability as compound 7, whereas compound 6 was less stable than compound 7. Compound 1 was the least stable of these compounds. Yoshida et al. concluded that the double bond configurations did not affect the stability, although the acyl group influenced the color stability (Yoshida et al., 1990). To conclude, compound 7 is converted to compound 6 by the elimination of a malonyl group upon exposure to light or air during storage. Compound 5 contains a malonyl group and is converted to compound 3a, which lacks a malonyl group. Compound 4 is the cis isomer of compound 7 and decreases in parallel with the decrease in compound 7; a similar change is observed for compounds 6 and $3 b$.

\subsection{Biosynthesis of anthocyanins in perilla}

Compounds 1 and 2 have a glucose moiety attached at the $3-O^{-}$and $5^{-} O$-positions, similar to compounds 3 to 7 . The biosynthetic pathway of anthocyanins in P. citriodora is predicted to be similar to that of $P$. frutescens, because the anthocyanins in these species are the same. In other plants, previous reports predicted that non-acylated anthocyanins were produced by the partial hydrolysis of acylated anthocyanins (Saito et al., 2008; Tatsuzawa et al., 2011). If partial hydrolysis were to occur in perilla, then compounds 1 and 2 would form from compounds 5 and 7 , while compounds 3, 4, 5, 6, and 7 would have formed as intermediates. If this were so, then the HPLC pattern of 
compounds 1 and 2 (e.g., pattern C (No. 5717)) would not have been observed. Accordingly, the biosynthetic pathway for compound 2 in $P$. citriodora appears to be involved in glycosylation at the 5- $O$-position and subsequent malonylation at the 6 '- $O$ position of the 5-O-glucose (Fig. 4). P. citriodora No. 87 harbored all seven species of anthocyanins when it was grown under natural sunlight (Fig. 1, pattern B). However, only compounds 1 and 2 were detected from No. 87 when it was grown under fluorescent lamps, and those of 1, 2, 4, and 7 were detected under UV-enhanced fluorescent lamps (data not shown). These experiences do not contradict results of previous studies showing that the expression of synthetic enzymes relevant to anthocyanins was induced by lighting (Saito and Yamazaki, 2002; Yamazaki et al., 1999), especially UV light (Nishimura et al., 2008; Nishimura et al., 2009). Although confirmatory experiments have not yet been performed, they may elucidate the biosynthetic pathway of cyanidins in No. 87 and indicate that compound 2 is formed from 1, and then 4 and 7 are formed afterwards (Fig. 4).

A biosynthetic pathway that generates compound 1 from cyanidin 3- $O$-glucose has been predicted in red perilla. A previous study showed that recombinant proteins in yeast extracts catalyzed the conversion of anthocyanidin $3-O$-glucosides into the corresponding anthocyanidin 3,5-O-diglucosides (Yamazaki et al., 1999). However, only $P$. citriodora contained compound 1 in the present study. Accordingly, some strains of $P$. citriodora may have an enzyme that catalyzes this pathway, namely, Cy3Glc 5GT, a 5$O$ glucosyltransferase specific to cyanidin $3-O$ glucoside. This suggests that wild species may have originally harbored the gene for Cy3Glc 5GT but that this gene, or its expression, was lost during evolution. Thereafter, different wild species that were unable to form cyanidin 3,5- $O$-diglucoside appeared, and cultivated species are likely amphidiploids of two wild species. Jánváry et al. (2009) reported a similar event for anthocyanins in Vitis vinifera: anthocyanins in Vitis species other than $V$. vinifera and 
most of their hybrids are glycosylated at the 3- $\mathrm{O}^{-}$and 5- $\mathrm{O}^{-}$-postions and form anthocyanin 3,5- $O$-diglucoside. However, some $V$. vinifera accumulate anthocyanin 3-O glucoside as a major anthocyanin and anthocyanin 3,5-O-diglucoside is detected in trace amounts. Genetic crossing experiments between $V$. vinifera and interspecific hybrids showed the presence of 5GT- $C h a$, which encodes an activated enzyme, and the allele 5GT-Dia. Site-directed mutagenesis and functional experiments showed that two mutations in 5GT-Cha render the 5GT inactive in $V$. vinifera (Jánváry et al., 2009). Anthocyanidin-3-O-glucoside acyltransferase (3AT) catalyzes the acylation of cyanidin 3-O-glucoside early in the biosynthesis of compounds 3 to 7 (Yonekura-Sakakibara et al., 2000). Comparison of the expression levels of $3 \mathrm{AT}$ showed that strains containing compounds 3 to 7 (Strain Nos. 79,5601, and 5645) had 3 to 17 times the expression levels of a strain containing compounds 1 to 7 (Strain No. 87) (Fig. 5). This result indicates that strains that accumulate compounds 3 to 7 contain 3AT, which catalyzes the acylation of $3-O$ glucose. In contrast, the strain that synthesizes compounds 1 to 7 (Strain No. 87) likely contains two enzymes that each catalyzes the acylation of cyanidin 3-O-glucoside to cyanidin 3-O-p-coumaryl and the glycosylation of cyanidin 3$O$ glucoside to cyanidin 3, 5- $O$-diglucoside. The expression level of 3AT in Strain No. 87, which co-expresses Cy3Glc 5GT, was low compared with the levels in strains that accumulate only compounds 3 to 7 , and Strain No. 87 . Therefore, the end product of both pathways is a high level of compound 7 .

Acylation of the $3-O$ glucose of compounds 3 to 7 is characteristic of perilla anthocyanins. Some acyltransferases catalyze the acylation of glucose. These compounds contain aliphatic acyl and aromatic acyl groups, the latter of which is likely mainly responsible for the co-pigmentation of anthocyanins. Molecular association formed by acyl groups and the cyanidin skeleton increases the stability of cyanidin compounds and prevents hydrolysis by hydrolase. Compound 2 is not acylated at the 3- 
$O$ glucose and is degraded by partial hydrolysis during storage, and is therefore probably less stable than compounds 3 to 7 , which have an acyl group at the $3-O$ glucose. Previous studies showed that compound 7, malonylshisonin, is the main perilla anthocyanin (Kondo et al., 1989; Yamazaki et al., 2003; Yoshida et al., 1990), in accordance with pattern $\mathrm{A}$ in the current study. Compound 7 is the most stable anthocyanin, is accumulated as the main component in perilla, is acylated at the $3-O$ and 5-O-glucose, and the acyl group of the $3^{-} \mathrm{O}$-glucose is aromatic.

Cultivated species were likely originally amphidiploids of two dominant wild species expressing acyltransferase (AT). The wild species $P$. citriodora was originally classified as three subspecies according to the anthocyanins present: a strain with only Cy3Glc 5GT and low-stability anthocyanins (No. 5717), a strain with AT and high-stability anthocyanins (No. 4931, etc.), and a strain with both Cy3Glc 5GT and AT (No. 87). Perhaps, during evolution, the strain encoding only Cy3Glc 5GT was evolutionarily uncompetitive because they could synthesize only low-stability anthocyanins, and thus strains expressing AT prospered.

\section{Conclusion}

Seven anthocyanins in perilla were identified in this study. Several reports have shown that many secondary metabolites are genetically controlled, consistent with the results of the present study. All red perilla ( $P$. frutescens) and most $P$. citriodora strains accumulate compounds 3 to 7 , only one strain of $P$. citriodora (No. 87) accumulated compounds 1 to 7, and another strain of P. citriodora (No. 5717) accumulated compounds 1 and 2 . In contrast, no foliar anthocyanins were detected in the green perilla $P$. frutescens, $P$. setoyensis, and $P$. hirtella. These results support the hypothesis that $P$. frutescens is an amphidiploid of two wild species, and P. citriodora may be an origin species. A putative biosynthetic pathway for perilla anthocyanins was 
described (Yamazaki et al., 1999). However, a strain that accumulated only compounds 1 and 2 was identified in this study, and therefore perilla likely expresses a 5- $O$ glucosyltransferase specific to cyanidin 3-O-glucoside. This strain accumulated seven compounds, and therefore likely has another pathway for synthesizing malonylshisonin as a main component, perhaps from compound 2 via compound 1 . These results provide clues to elucidating the biosynthetic pathway for anthocyanins, the evolution of perilla, and may facilitate phylogenetic systematics. Acylation increases the stability of anthocyanins (Matsufuji et al., 2007). The analysis of anthocyanin species and their concentrations in perilla herbs showed that drying and exposure to light during storage change the deacylation of anthocyanins.

\section{Experimental}

\subsection{General}

All chemical reagents and solvents were purchased from Nacalai Tesque Inc, Toyobo Co., Ltd., or Qiagen, and were of HPLC grade or otherwise the highest grade available. HPLC analyses were performed using an L-2000 series (Hitachi) or LC2000 series (JASCO) system with a UV-Vis detector and a $150 \times 4.6 \mathrm{~mm}$ i.d. COSMOSIL Cholester column (Nacalai Tesque). Preparative-scale chromatographic separations were carried out using a Prominence series (Shimadzu) system with a UV-Vis detector and $250 \times 20$ mm i.d. COSMOSIL Cholester column (Nacalai Tesque). LC-MS data were obtained using a Prominence series LCMS-IT-TOF system equipped with an ESI interface (Shimadzu). ${ }^{1} \mathrm{H}$ and ${ }^{13} \mathrm{C}$ NMR data were obtained on a JNM-ECA500KP $\left({ }^{1} \mathrm{H}\right.$ at 500 $\mathrm{MHz},{ }^{13} \mathrm{C}$ at $\left.125 \mathrm{MHz}\right)$ spectrometer (JEOL) in $3 \% d$-TFA $\mathrm{CD}_{3} \mathrm{OD}$ at $20^{\circ} \mathrm{C}$. Quantitative real-time PCR analyses were performed using an Applied Biosystems 7300 Real Time PCR System (Applied Biosystems).

\subsection{Plant materials}


Perilla plants (Table 2a) were grown at the Experimental Station for Medicinal Plant Research, Graduate School of Pharmaceutical Science, Kyoto University. All plants were bred and kept as pure lines through repeated self-pollination by covering flowers with paper pollinating bags before the flowers opened and collecting the mature seeds. Fresh perilla herb leaves were collected from a botanical garden in Nagoya (No. 1-3, Table 2b). Dried commercially available perilla herb leaves were kindly donated by Shoyakuhinshitusyudankai or purchased from Daiko Shoyaku (No. 4-21, Table 2b). Voucher specimens of dried perilla herb leaves were deposited in the herbarium of the Experimental Station for Medicinal Plant Research (Specimen numbers: EST-5017 to 5034). Natural dyes made from red perilla were kindly provided by Nichinou Kagaku Kougyou Co.

\subsection{HPLC analysis}

Samples (approximately $30 \mathrm{mg}$ ) of fresh leaves (Table 2a) and dried leaves (Table 2b) were extracted with a $0.5 \mathrm{~mL}$ mixed solution of $\mathrm{AcOH}-\mathrm{H}_{2} \mathrm{O}-\mathrm{MeOH}(1: 10: 9)$ for $48 \mathrm{~h}$ at $4{ }^{\circ} \mathrm{C}$ in the dark. The extract was filtered through a $0.45 \mu \mathrm{m}$ filter. Anthocyanins were analyzed by HPLC. A sample $(15 \mu \mathrm{L})$ of the filtered extract was injected onto an analytical column (COSMOSIL Cholester, $150 \times 4.6 \mathrm{~mm}$ i.d.) maintained at $30{ }^{\circ} \mathrm{C}$. The mobile phase was composed of $0.1 \% \mathrm{TFA}(\mathrm{v} / \mathrm{v})$ in water (eluent A) and $0.1 \% \mathrm{TFA}(\mathrm{v} / \mathrm{v})$ in acetonitrile (eluent B) and was delivered at a flow rate of $0.5 \mathrm{~mL} / \mathrm{min}$. The gradient program was $15 \%$ B (0 min), 15-48\% B (0-38 min), then held for 12 min before returning to the initial conditions. Total run time was $50 \mathrm{~min}$. Detection was at 528 $\mathrm{nm}$.

\subsection{Preparation and identification of anthocyanins}

Fresh leaves of Strain No. 5717 (73.7 g) and Strain No. 5601 (34.5 g) were collected, cut, and extracted twice with $500 \mathrm{~mL}$ (Strain No. 5717) or $400 \mathrm{~mL}$ (Strain No. 5601) of $3 \%$ TFA (v/v) in water for $48 \mathrm{~h}$ at $4{ }^{\circ} \mathrm{C}$ in the dark. The water extract was concentrated 
under reduced pressure and the resulting residue was purified by Amberlite XAD-7 column chromatography. The column was washed with $1 \%$ TFA (v/v) in water and the anthocyanins were eluted with $1 \% \mathrm{TFA}(\mathrm{v} / \mathrm{v})$ in methanol. The eluate was concentrated under reduced pressure and dried by lyophilization. The crude anthocyanin extract was dissolved 1\% TFA (v/v) in water and then centrifuged at $13000 \mathrm{rpm}$ for $1 \mathrm{~min}$. The supernatant $(2 \mathrm{~mL})$ was injected onto a preparative column (COSMOSIL Cholester, 250 $\times 20 \mathrm{~mm}$ i.d.) maintained at $30{ }^{\circ} \mathrm{C}$. The mobile phase was composed of $0.1 \%$ TFA (v/v) in water (eluent $\mathrm{A})$ and $0.1 \%$ TFA (v/v) in acetonitrile (eluent B) and was delivered at a flow rate of $8.0 \mathrm{~mL} / \mathrm{min}$. The isocratic program was $22 \% \mathrm{~B}$ held for $75 \mathrm{~min}$ for the extract from No. 5601 and 17\% B held for 45 min for the extract from No. 5717. Detection was at $528 \mathrm{~nm}$. Compounds 1 and 2 were obtained from No. 5717, and compounds 3 to 7 were obtained from No. 5601. Compounds were identified by LC-MS and NMR analysis.

\subsection{Transcript abundance analysis by quantitative real-time PCR (qPCR)}

Total RNA was isolated from fresh young leaves of Strain Nos. 79, 87,. 5601, and 5645 using an RNeasy Plant Mini Kit (Qiagen), and reverse transcribed with the primer add2 \{5'-CCACGCGTCGACTACTTTTTTTTTTTTTTT-3'\} using ReverTra Ace (Toyobo). The synthesized cDNA was used as a template for qPCR. PCR was performed using an Applied Biosystems 7300 Real Time PCR System (Applied Biosystems) in a reaction mixture containing THUNDERBIRD SYBR qPCR Mix (Toyobo), 50X ROX reference dye, and $0.3 \mu \mathrm{M}$ forward and reverse primers using the following cycling parameters: $95^{\circ} \mathrm{C}$ for $60 \mathrm{~s}$, followed by 40 cycles of $95{ }^{\circ} \mathrm{C}$ for $15 \mathrm{~s}, 52{ }^{\circ} \mathrm{C}$ for $15 \mathrm{~s}, 72^{\circ} \mathrm{C}$ for $60 \mathrm{~s}$, then melting curve analysis at $95^{\circ} \mathrm{C}$ for $15 \mathrm{~s}, 60^{\circ} \mathrm{C}$ for $60 \mathrm{~s}, 95^{\circ} \mathrm{C}$ for $15 \mathrm{~s}, 60{ }^{\circ} \mathrm{C}$ for $15 \mathrm{~s}$. Transcript abundance was normalized to the transcript abundance of the histone H2A gene and was calculated from three technical replicates. Relative transcript abundances were calibrated against the transcript abundance of control samples and 
were calculated using the Comparative $\mathrm{Ct}$ method. The primer pairs used for qPCR were as follows: histone (product size: 141bp): 5'-CCGTTATCTGAAGAAAGGTCGCTAT3') (forward) and 5'ATCCTGTTCTTCTTGTTGTCTCGTG-3') (reverse); 3AT (product size: 134bp): 5’-CCCAGATTCAATTCATCTCTCCTCT-3') (forward) and 5'GTCGTAGATCGATCGGAATCAAGAA-3') (reverse).

\section{Acknowledgements}

We are very grateful to Shoyakuhinshitusyudankai and Nichinou Kagaku Kougyou Co. for providing the plant samples.

Funding source declaration: This work was partially supported by a Grant-in-Aid for Young Scientists (B) from the Japan Society for the Promotion of Science (Grant Number 16K18902) and Kinjo Gakuin University Research Grant. Conflict of interest statement: None.

\section{References}

Charron, C.S., Clevidence, B.A., Britz, S.J., Novotny, J.A., 2007. Effect of dose size on bioavailability of acylated and nonacylated anthocyanins from red cabbage (Brassica oleracea L. var. capitata). J. Agric. Food. Chem. 55, 5354-5362.

Gong, Z., Yamazaki, M., Sugiyama, M., Tanaka, Y., Saito, K., 1997. Cloning and molecular analysis of structural genes involved in anthocyanin biosynthesis and expressed in a forma-specific manner in Perilla frutescens. Plant Molecular Biology. 35, 915-927.

Goto, T., Hoshino, T., Ohba, M., 1976. Stabilization effect of neutral salts on anthocyanins: flavylium salts, anhydrobases and genuine anthocyanins. Agric. Biol. Chem. 40, 1593-1596. 
Goto, T., Kondo, T., Tamura, H., Takase, S., 1983. Structure of malonylawobanin, the real anthocyanin present in blue-colored flower petals of Commelina communis. Tetrahedron Lett. 24, 4863-4866.

He, F., Mu, L., Yan, Guo-L., Liang, Na-N., Pan, Qui-H., Wang, J., Reeves, M. J., Duan, Chang-Q., 2010. Biosynthesis of anthocyanins and their regulation in colored grapes. Molecules 15, 9057-9091.

Honda, G., 1996. Genetic control of essential oil components in Perilla. FFI. J. 169, 915.

Honda, G., Koezuka, Y., Tabata, M., 1990. Genetic studies of fruits color and hardness in Perilla frutescens. Jap. J. Breed. 40, 469-474.

Hosokawa, K., Fukunaga, Y., Fukushi, E., Kawabata, J., 1995. Acylated anthocyanins from red Hyacinthus orientalis. Phytochemistry 39, 1437-1441.

Ito, M., Kato, H., Oka, Y., Honda, G., 1998. Phylogenetic analysis of Japanese Perilla species by using DNA polymorphisms. Natural Medicines 52, 248-252.

Ito, M., Toyoda, M., Honda, G., 1999a. Chemical composition of the essential oil of Perilla frutescens. Natural Medicines 53, 32-36.

Ito, M., Toyoda, M., Honda, G., 1999b. Essential oil composition of hybrids and amphidiploids of Japanese wild perilla Natural Medicines 53, 118-122.

Ito, M., Toyoda, M., Kamakura, S., Honda, G., 2002. A new type of essential oil from Perilla frutescens from Thailand. J. Essent. Oil Res. 14, 416-419.

Jánváry, L., Hoffmann, T., Pfeiffer, J., Hausmann, L., Töpfer, R., Fischer, T-C., Schwab, W., 2009. A double mutation in the anthocyanin 5- $O$-glucosyltransferase gene disrupts enzymatic activity in Vitis vinifera L. J. Agric. Food Chem. 57, 35123518.

Kitada, C., Gong, Z., Tanaka, Y., Yamazaki, M., Saito, K., 2001. Differential expression of two cytochrome P450s involved in the biosynthesis of flavones and anthocyanins in 
chemo-varietal forms of Perilla frutescens. Plant Cell Physiol. 42, 1338-1344.

Koezuka, Y., Honda, G., Sakamoto, Y., Tabata M., 1985. Genetic control of anthocyanin production in Perilla frutescens. Syoyakugaku Zasshi 39, 228-231.

Koezuka, Y., Honda, G., Tabata, M., 1986. Genetic control of the chemical composition of volatile oils in Perilla frutescens. Phytochemistry 25, 859-863.

Kondo, T., Tamura, H., Yoshida, K., Goto, T., 1989. Structure of malonylshisonin, a genuine pigment in purple leaves of Perilla ocimoides L. var. crispa Benth. Agric. Biol. Chem. 53, 797-800.

Masumoto, N., Korin, M., Ito, M., 2010. Geraniol and linalool synthases from wild species of perilla. Phytochemistry 71, 1068-1075.

Matsufuji, H., Kido, H., Misawa, H., Yaguchi, J., Otsuki, T., Chino, M., Takeda, M., Yamagata, K., 2007. Stability to light, heat, and hydrogen peroxide at different $\mathrm{pH}$ values and DPPH radical scavenging activity of acylated anthocyanins from red radish extract. J. Agric. Food Chem. 55, 3692-3701.

Matsune, T., Koike, A., Kato, K., Kawanobu, S., Ino, I., Yamaguchi, M., 1997. Characterization of anthocyanin acylation enzyme on purple leaves of shiso (Perilla ocimoides L. var. crispa Benth). Engei Gakkai Zasshi 66, 92-93.

Nishimura, T., Ohyama, K., Goto, E., Inagaki, N., 2009. Concentrations of perillaldehyde, limonene, and anthocyanin of Perilla plants as affected by light quality under controlled environments. Scientia Horticulturae 122, 134-137.

Nishimura, T., Ohyama, K., Inagaki, N., Morota, T., Goto, E., 2008. Ultraviolet-B radiation suppressed the growth and anthocyanin production of Perilla plants grown under controlled environments with artificial light. Acta Horticulturae 797, 425-429.

Saito, K., Yamazaki, M., 2002. Biochemistry and molecular biology of the late-stage of biosynthesis of anthocyanin: lessons from Perilla frutescens as a model plant. New Phytol 155, 9-23 
Saito, N., Tatsuzawa, F., Suenaga, E., Toki, K., Shinoda, K., Shigihara, A., Honda, T., 2008. Tetra-acylated cyanidin 3-sophoroside-5-glucosides from the flowers of Iberis umbellate L. (Cruciferae). Phytochemistry 69, 3139-3150.

Shirai, M., 1930. Tochu kokuyaku honzokomoku so-bu 14kan. Shunyodo, Tokyo, pp. 634.

Suzuki, H., Nakayama, T., Yonekura-Sakakibara, K., Fukui, Y., Nakamura, N., Nakao, M., Tanaka, Y., Yamaguchi, M., Kusumi, T., Nishino, T., 2001. Malonyl-CoA: Anthocyanin 5-O-glucoside-6"'-O-malonyltransferase from scarlet sage (Salvia splendes) flowers. J. Biol. Chem. 276, 49013-49019.

Tamura, H., Kondo, T., Kato, Y., Goto, T., 1983. Structures of a succinyl anthocyanin and a malonyl flavone, two constituents of the complex blue pigment of cornflower Centaurea cyanus. Tetrahedron Lett. 24, 5749-5752.

Tatsuzawa, F., Miyoshi, K., Yukawa, T., Shinoda, K., Toki, K., Saito, N., Shigihara, A., Honda, T., 2011. Malonylated anthocyanidin 3, 5-diglucosides in the flowers of the genus Disa. (Orchidaceae). Biochem. Syst. Ecol. 39, 220-224.

The Ministry of Health, Labour and Welfare, 2016. The Japanese Pharmacopoeia, seventeenth ed. The Ministry of Health, Labour and Welfare, Tokyo, p. 1843. Yamazaki, M., Gong, Z., Fukuchi-Mizutani, M., Fukui, Y., Tanaka, Y., Kusumi, T., Saito, K., 1999. Molecular cloning and biochemical characterization of a novel anthocyanin 5-O-glucosyltransferase by mRNA differential display for plant forms regarding anthocyanin. J. Biol. Chem. 274, 7405-7411.

Yamazaki, M., Nakajima, J., Yamanashi, M., Sugiyama, M., Makita, Y., Springob, K., Awazuhara, M., Saito, K., 2003. Metabolomics and differential gene expression in anthocyanin chemo-varietal forms of Perilla frutescens. Phytochemistry 62, 987-995.

Yokoi, M., Saito, N., Kawabata, Y., Suzuki, S., Hirabayashi, H., 1979. Relation of quantitative flower color measurement to the flower color in rose cultivars. Chiba 
Daigaku Engeigakubu Gakujutsu Hokoku. 26, 1-8.

Yonekura-Sakakibara K., Tanaka Y., Fukuchi-Mizutani M., Fujiwara H., Fukui Y., Ashikari T., Murakami Y., Yamaguchi M., Kusumi T., 2000. Molecular and biochemical characterization of a novel hydroxycinnamoyl-CoA: anthocyanin 3-O glucoside-6"- $O$-acyltransferase from Perilla frutescens. Plant Cell Physiol. 41, 495502.

Yoshida, K., Kondo, T., Kameda, K., Goto, T., 1990. Structure of anthocyanins isolated from purple leaves of Perilla ocimoides L. var. crispa Benth and their isomerization by irradiation of light. Agric. Biol. Chem. 54, 1745-1751.

Yu, He-Ci., Kosuna, K., Haga, M., 1997. Perilla: The Genus Perilla. Taylor \& Francis, Amsterdam., pp. 149-170. 
Figure legends

Fig. 1 HPLC chromatograms of perilla anthocyanins

Fig. 2 Chemical structures of compounds

Fig. 3 Relationship between anthocyanin content and collection date

Fig. 4 Putative biosynthetic pathway of perilla anthocyanins

Cy3Glc 5GT: 5-O-glucosyltransferase specific to cyanidin 3-O-glucoside

3AT: Anthocyanin 3-O-glucoside acyltransferase

MT: Malonyl transferase

UA5GT: UDP-glucose-dependent anthocyanin 5-O-glucosyltransferase

AT: Acyltransferase

$-\rightarrow$ : Putative pathway

Fig. 5 Comparison of the expression levels of acyltransferase as measured by real-time PCR 
Table 1 Perilla anthocyanin patterns and essential oil types

\begin{tabular}{|c|c|c|c|}
\hline Species name & Strain number & $\begin{array}{l}\text { Anthocyanin } \\
\text { pattern }\end{array}$ & $\begin{array}{l}\text { Essential oil } \\
\text { type }\end{array}$ \\
\hline$P$. frutescens (green leaf) & 10 & $\mathrm{D}$ & PP-em \\
\hline P. frutescens (green leaf) & 12 & $\mathrm{D}$ & PP-m \\
\hline$P$. frutescens (green leaf) & 1841 & $\mathrm{~A}$ & $\mathrm{EK}$ \\
\hline$P$. frutescens (green leaf) & 1864 & $\mathrm{D}$ & PL \\
\hline$P$. frutescens (green leaf) & 5598 & $\mathrm{D}$ & $\mathrm{PT}$ \\
\hline$P$. frutescens (green leaf) & 5808 & $\mathrm{D}$ & $\mathrm{PK}$ \\
\hline$P$. frutescens (green leaf) & 79 & $\mathrm{~A}$ & EK \\
\hline P. frutescens (red leaf) & 6 & $\mathrm{~A}$ & $\mathrm{PK}$ \\
\hline P. frutescens (red leaf) & 25 & $\mathrm{~A}$ & PP-emd \\
\hline P. frutescens (red leaf) & 32 & $\mathrm{~A}$ & $\mathrm{PA}$ \\
\hline$P$. frutescens (red leaf *) & 63 & $\mathrm{~A}$ & PK \\
\hline P. frutescens (red leaf) & 5343 & $\mathrm{~A}$ & $\mathrm{PA}$ \\
\hline P. frutescens (red leaf) & 5645 & $\mathrm{~A}$ & $\mathrm{PA}$ \\
\hline P. frutescens (red leaf) & 5647 & $\mathrm{~A}$ & PA \\
\hline P. frutescens (red leaf) & 5807 & $\mathrm{~A}$ & PA \\
\hline P. citriodora & 87 & $\mathrm{~B}$ & $\mathrm{C}$ \\
\hline P. citriodora & 4931 & $\mathrm{~A}$ & $\mathrm{C}$ \\
\hline P. citriodora & 4935 & $\mathrm{~A}$ & $\mathrm{PK}$ \\
\hline P. citriodora & 5321 & $\mathrm{~A}$ & PP-em \\
\hline P. citriodora & 5498 & $\mathrm{~A}$ & $\mathrm{C}$ \\
\hline P. citriodora & 5601 & $\mathrm{~A}$ & $\mathrm{PP}, \mathrm{PT}$ \\
\hline
\end{tabular}




\begin{tabular}{lccc} 
P. citriodora & 5712 & $\mathrm{~A}$ & $\mathrm{PK}$ \\
P. citriodora & 5717 & $\mathrm{C}$ & $\mathrm{C}$ \\
\hline P. setoyensis & 5031 & $\mathrm{D}$ & $\mathrm{SF}$ \\
$P$. setoyensis & 5640 & $\mathrm{D}$ & $\mathrm{SF}$ \\
\hline P. hirtella & 4993 & $\mathrm{D}$ & $\mathrm{PK}$ \\
$P$. hirtella & 5042 & $\mathrm{D}$ & PP-e
\end{tabular}

Anthocyanin patterns are corresponding to Fig. 1.

*: bicolor 
Table 2a Perilla species names and strain no.

\begin{tabular}{ll}
\hline Species name & Strain No. \\
\hline P. frutescens (green leaf) & $10,12,1841,1864,5598,5808,5343$ \\
P. frutescens (red leaf) & $3,6,9,25,32,63^{*}, 79,5645,5647,5807$ \\
P. citriodora & $87,4931,4935,5321,5498,5601,5717$ \\
P. setoyensis & 5031,5640 \\
P. hirtella & 4993,5042 \\
\hline *: bicolor) &
\end{tabular}

Table $2 \mathrm{~b}$ Characteristics of perilla herbs and dye samples

\begin{tabular}{lllll}
\hline Sample & Collection & Collection & Collection or & Product form \\
No. & country & site & manufacture date & \\
\hline 1 & Japan & Nagoya & 2015.09 & fresh \\
2 & Japan & Nagoya & 2015.09 & fresh \\
3 & Japan & Nagoya & 2015.09 & fresh \\
4 & Japan & Miyazaki & 2014.11 & dry/cut \\
5 & Japan & Shizuoka & 2013.08 & dry/cut \\
6 & China & Liaoning & 2015.05 & dry/cut \\
7 & China & Liaoning & 2013.07 & dry/cut \\
8 & China & Hebei & 2015.09 & dry/cut \\
9 & China & Hebei & 2015.09 & dry/cut \\
10 & China & Hebei & 2011.05 & dry/cut \\
11 & China & Hebei & 2014.04 & dry/cut \\
12 & China & Hebei & 2014.12 & dry/cut \\
13 & China & Hebei & 2015.12 & dry/cut \\
\hline
\end{tabular}




$\begin{array}{lllll}14 & \text { China } & \text { Anhui } & 2012.02 & \text { dry/cut } \\ 15 & \text { China } & \text { Fujian } & 2015.09 & \text { dry/cut } \\ 16 & \text { China } & \text { Guangdong } & 2015.02 & \text { dry/cut } \\ 17 & \text { China } & - & - & \text { dry/cut } \\ 18 & \text { China } & - & 2015.02 & \text { dry/cut } \\ 19 & \text { China } & - & 2015.01 & \text { dry/cut } \\ 20 & \text { China } & - & 2014.03 & \text { dry/cut } \\ 21 & \text { China } & - & 2015.12 & \text { dry/cut } \\ 22 & \text { Japan } & - & - & \text { liquid preparation }\end{array}$

(- : details unknown) 
Table $3{ }^{1} \mathrm{H}$ and ${ }^{13} \mathrm{C}$ NMR data of peak 2 in $3 \% d \Gamma F A-\mathrm{CD}_{3} \mathrm{OD}$

\begin{tabular}{|c|c|c|c|}
\hline & \multirow[t]{2}{*}{ Position } & \multicolumn{2}{|c|}{ Peak 2} \\
\hline & & $\delta_{\mathrm{C}}$ & $\delta_{\mathrm{H}}(J$ in $\mathrm{Hz})$ \\
\hline \multirow[t]{15}{*}{ Anthocyanidin } & 2 & 164.9 & \\
\hline & 3 & 146.7 & \\
\hline & 4 & 135.9 & $9.14 \mathrm{~s}$ \\
\hline & 5 & 157.1 & \\
\hline & 6 & 106.0 & $7.03 \mathrm{~d}(2.0)$ \\
\hline & 7 & 169.4 & \\
\hline & 8 & 97.3 & $7.10 \mathrm{br} \mathrm{s}$ \\
\hline & 9 & 153.9 & \\
\hline & 10 & 113.3 & \\
\hline & $1^{\prime}$ & 121.1 & \\
\hline & $2^{\prime}$ & 118.5 & $8.09 \mathrm{~d}(2.5)$ \\
\hline & $3^{\prime}$ & 147.6 & \\
\hline & $4^{\prime}$ & 156.6 & \\
\hline & $5^{\prime}$ & 117.6 & $7.05 \mathrm{~d}(9.0)$ \\
\hline & $6^{\prime}$ & 129.1 & $8.36 \mathrm{dd}(9.0,2.5)$ \\
\hline \multirow[t]{7}{*}{ Glucose A } & 1 & 104.0 & $5.30 \mathrm{~d}(8.0)$ \\
\hline & 2 & 74.7 & $3.69 \mathrm{dd}(8.5,6.5)$ \\
\hline & 3 & 78.3 & $3.53 \mathrm{t}(9.0)$ \\
\hline & 4 & 71.3 & 3.40 t (9.0) \\
\hline & 5 & 78.9 & $3.62 \mathrm{~m}$ \\
\hline & $6 a$ & & $3.71 \mathrm{dd}(12.0,7.0)$ \\
\hline & $6 \mathrm{~b}$ & & $3.97 \mathrm{dd}(12.0,2.5)$ \\
\hline
\end{tabular}




$\begin{array}{llll}\text { Glucose B } & 1 & 102.3 & 5.18 \mathrm{~d}(8.1) \\ & 2 & 74.4 & 3.69 \mathrm{dd}(8.5,6.5) \\ & 3 & 77.5 & 3.56 \mathrm{t}(9.0) \\ & 4 & 71.3 & 3.45 \mathrm{t}(9.0) \\ & 5 & 75.8 & 3.80 \mathrm{ddd}(12.0,7.0,2.5) \\ & 6 \mathrm{a} & & 4.34 \mathrm{dd}(12.0,6.5) \\ & 6 \mathrm{~b} & 65.2 & 4.55 \mathrm{dd}(12.0,2.5) \\ \text { Malonic acid } & 1 & 168.6 & \\ & 2 & 39.4 & 3.42 \mathrm{~s} \\ & 3 & 169.5 & \\ & & & \end{array}$




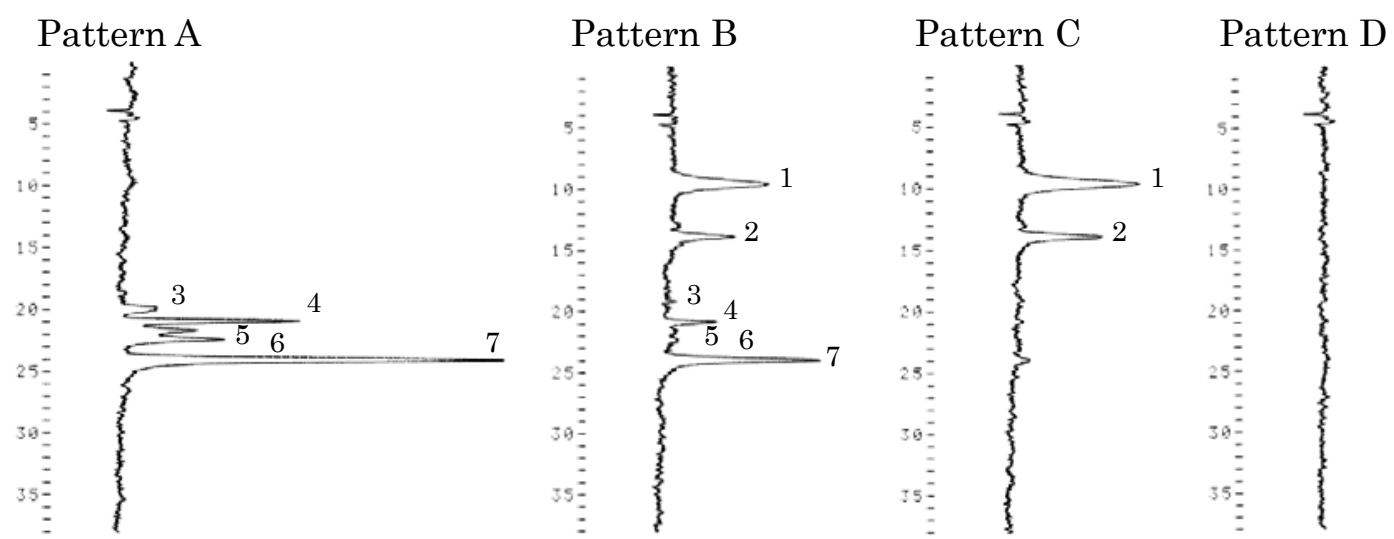

Fig. 2 HPLC chromatograms of perilla anthocyanins 


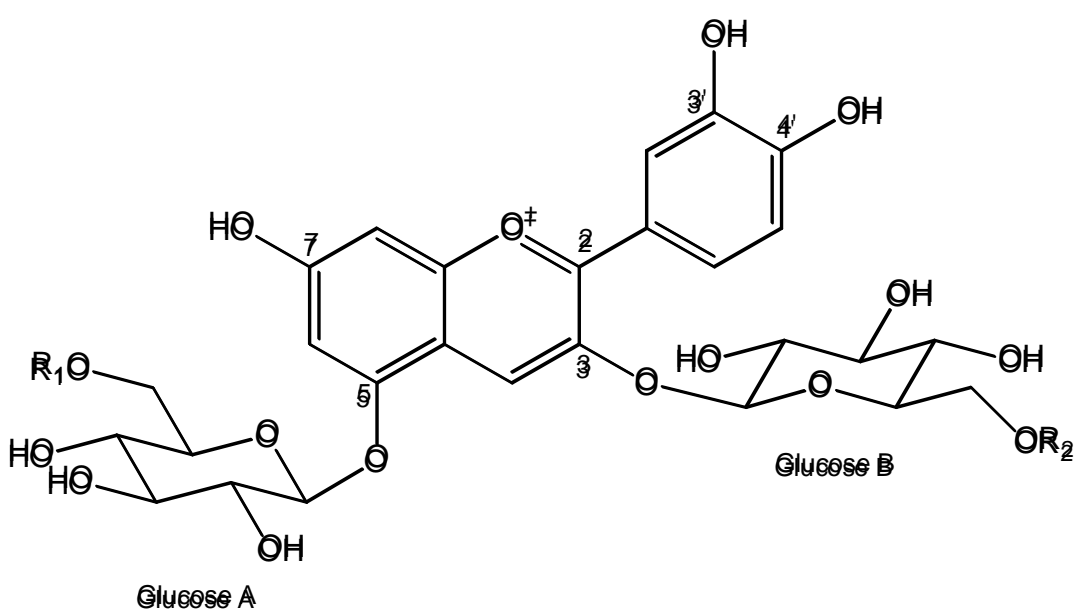

\begin{tabular}{|c|c|c|c|}
\hline Peak & Compound & $\mathrm{R}_{1}$ & $\mathrm{R}_{2}$ \\
\hline 1 & Cyanidin 3,5-O-diglucoside & $\mathrm{H}$ & $\mathrm{H}$ \\
\hline 2 & Cyanidin 3-O-glucoside-5-O-malonylglucoside & malonyl & $\mathrm{H}$ \\
\hline $3 a$ & Cyanidin 3-O-caffeylglucoside-5-O-glucoside & $\mathrm{H}$ & $\operatorname{trans} s^{-c a f f e y l}$ \\
\hline $3 b$ & cis-Shisonin & $\mathrm{H}$ & cis-p-coumaryl \\
\hline 4 & Malonyl-cis-Shisonin & malonyl & cis-p-coumaryl \\
\hline 5 & Cyanidin 3-O-caffeylglucoside-5-O-malonylglucoside & malonyl & trans-caffeyl \\
\hline 6 & Shisonin & $\mathrm{H}$ & trans-p-coumaryl \\
\hline 7 & Malonylshisonin & malonyl & trans-p-coumaryl \\
\hline
\end{tabular}

Fig. 2 Chemical structure of compounds 


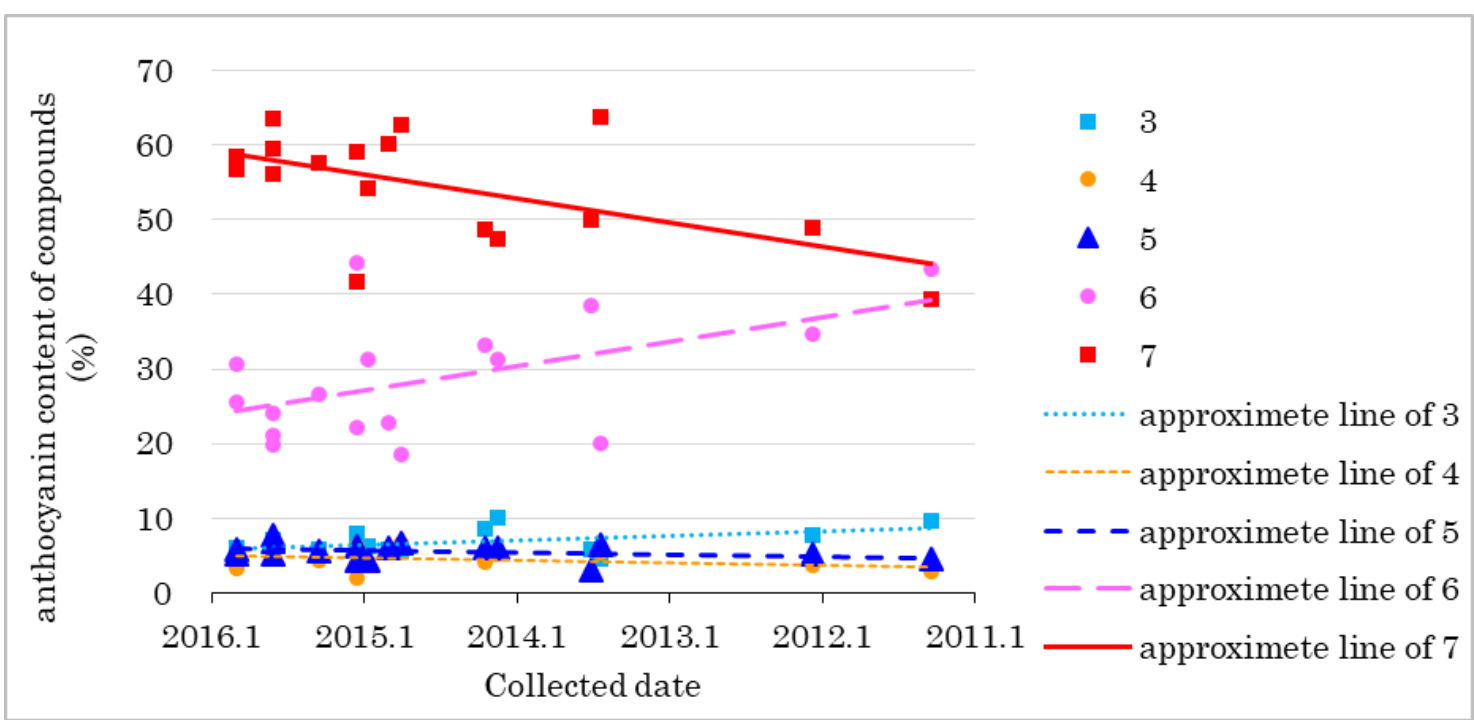

Fig. 3 Relationship between anthocyanin content and collected date 
<smiles>COc1cc2c(OC(C)C)cc(O)cc2[o+]c1-c1ccc(O)c(O)c1</smiles><smiles>Oc1cc(OC2CCCCC2)c2cc(OC3CCCCC3)c(-c3ccc(O)c(O[14C]4CCCCC4)c3)[o+]c2c1</smiles>

Compound 1

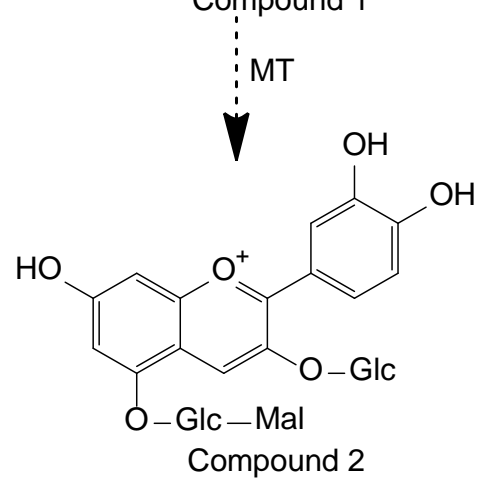

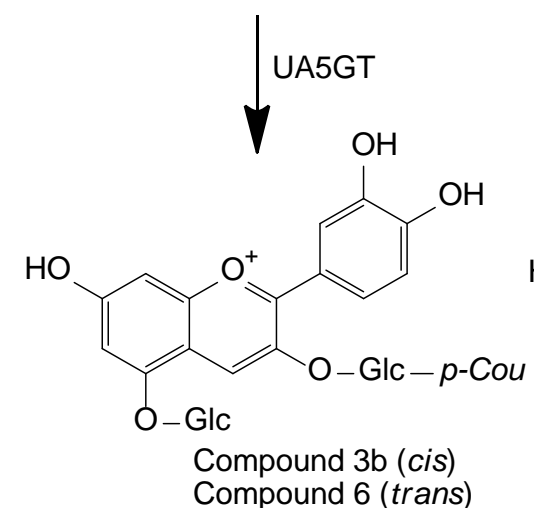<smiles>CC(C)[C@@H](C)Br</smiles>

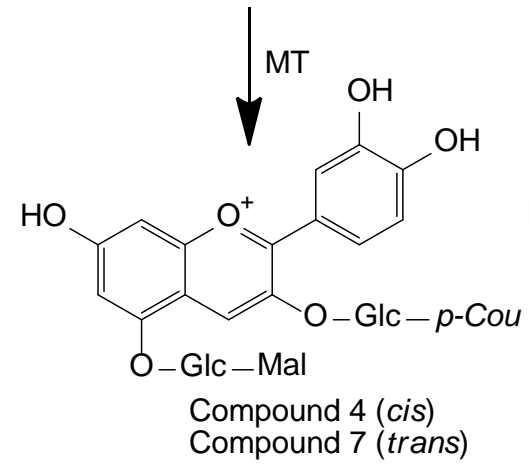

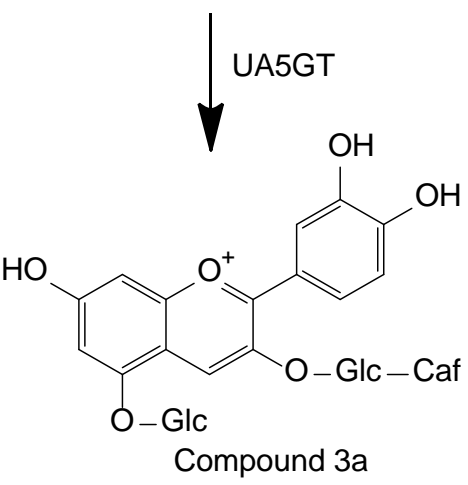

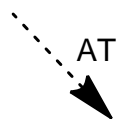

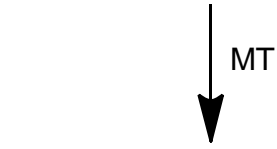

$\mathrm{HO}$<smiles>[M][Si]([O-])([O-])OOc1cc2cc(C)ccc2[o+]c1-c1ccc(O)c(C)c1</smiles>
Compound 5

Fig. 4 Putative biosynthetic pathway of perilla anthocyanins

Cy3Glc 5GT: 5- $O$-glucosyltransferase specific to cyanidin 3- $O$-glucoside 3AT: Anthocyanin 3-O-glucoside acyltransferase MT: Malonyl transferase

UA5GT: UDP-glucose-dependent anthocyanin 5- $O$-glucosyltransferase AT: Acyltransferase

$\rightarrow$ : Putative pathway 


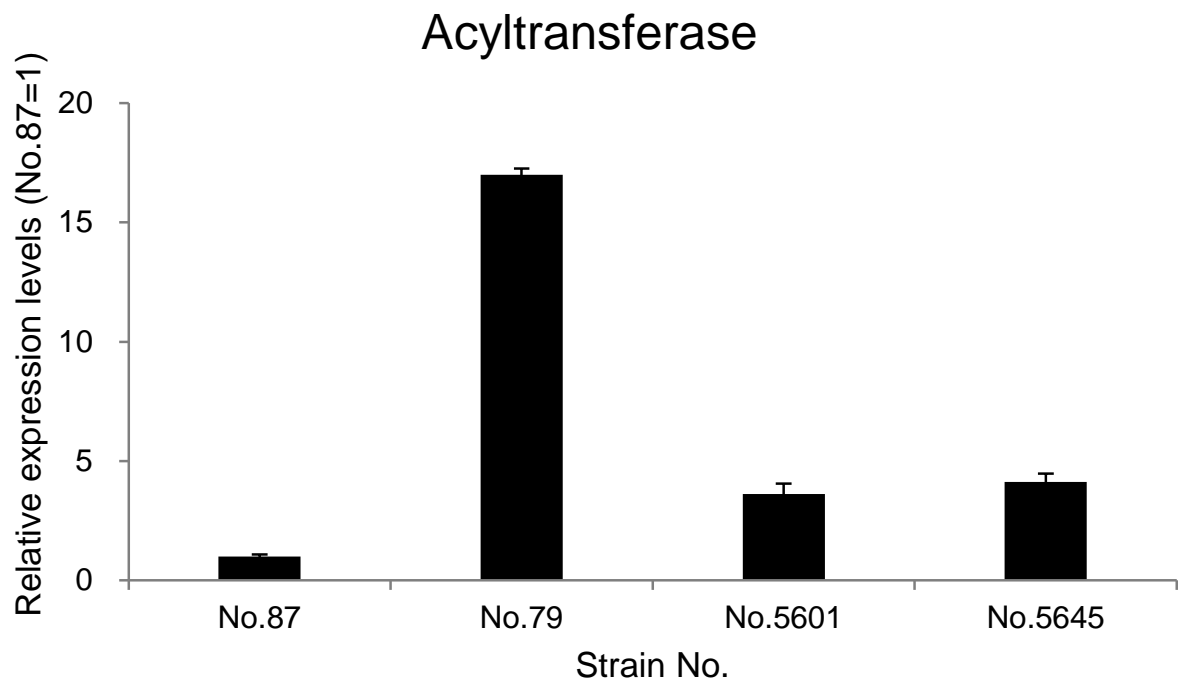

Fig. 5 Comparison of expression levels of acyltransferase measured by Real-time PCR 